\title{
Des classes bilingues dans l'académie de Strasbourg
}

\author{
Roger Schumacher
}

\section{(2) OpenEdition}

12 Journals

Édition électronique

URL : http://journals.openedition.org/ries/2956

DOI : 10.4000/ries.2956

ISSN : 2261-4265

Éditeur

Centre international d'études pédagogiques

Édition imprimée

Date de publication : 1 mars 1998

Pagination : 89-95

ISBN : 9771254459005

ISSN : $1254-4590$

\section{Référence électronique}

Roger Schumacher, " Des classes bilingues dans l'académie de Strasbourg », Revue internationale d'éducation de Sèvres [En ligne], 17 | 1998, mis en ligne le 19 avril 2013, consulté le 30 avril 2019. URL : http://journals.openedition.org/ries/2956 ; DOI : 10.4000/ries.2956

Ce document a été généré automatiquement le 30 avril 2019.

(ㄷ) Tous droits réservés 


\title{
Des classes bilingues dans l'académie de Strasbourg
}

\author{
Roger Schumacher
}

\section{Dates et événements fondateurs}

1 Pour comprendre l'actuelle problématique de l'enseignement bilingue à l'école en Alsace, il convient de prendre en compte l'évolution historique et linguistique de cette région depuis 1870 .

2 En 1870, seules les couches supérieures de la société alsacienne connaissaient suffisamment le français pour le parler couramment. Après l'annexion de l'Alsace à l'Empire allemand, l'introduction de l'allemand comme langue unique d'enseignement a contribué à faire régresser les efforts de francisation qui commençaient à se faire sentir avant 1870 .

3 En 1918, hormis certains milieux de la bourgeoisie qui pratiquaient le français, la grande majorité de la population était de langue et de culture germaniques.

4 Après le retour à la France, les autorités ont désigné la langue française comme langue exclusive d'enseignement en Alsace et les difficultés d'ordre linguistique furent grandes pour les instituteurs qui n'avaient pas été formés pour enseigner en français. Des cours de français furent organisés à leur intention et un grand nombre d'enseignants d'autres départements français furent envoyés en Alsace. Mais dès 1920, des députés alsaciens ont demandé une réforme linguistique permettant d'utiliser l'allemand comme vecteur d'enseignement au cours des premières années de la scolarité et ne prévoyant l'introduction du français que plus tard.

5 En 1920, la pression fut telle que le recteur Charléty dut accepter un compromis. Les circulaires du 15 janvier 1920 permettaient de commencer l'enseignement de l'allemand au début de la quatrième année scolaire et, au cours du mois d'octobre 1920, l'enseignement de l'allemand fut autorisé dès la troisième année scolaire à raison de trois 
heures hebdomadaires ajoutées à quatre heures d'éducation religieuse dispensées en allemand ou en dialecte.

6 En 1927, les revendications aboutirent à la circulaire du recteur Pfister du 31 janvier qui allait réglementer l'enseignement de l'allemand dans les écoles primaires. Elle prévoyait les dispositions suivantes :

- l'introduction de l'allemand pendant la deuxième année scolaire, à raison de deux heures par semaine, puis trois heures par semaine à partir de la troisième année scolaire ;

- l'introduction d'un épreuve d'allemand au certificat de fin d'études primaires ;

- l'éducation religieuse continuait à être dispensée en allemand, à raison de quatre heures par semaine.

7 En 1939, on estimait que la moitié de la population alsacienne était en mesure de s'exprimer en français, alors que la connaissance de l'allemand se maintenait et que le dialecte alsacien demeurait la langue parlée d'usage courant. Cette circulaire du recteur Pfister avait entraîné un certain apaisement dans la question linguistique.

8 Pendant l'annexion nazie de 1940 à 1945, la seule langue autorisée dans l'enseignement fut l'allemand standard, le français étant strictement interdit.

9 En 1945, après la Libération, la réglementation de 1927 n'a pas été remise en vigueur et l'enseignement de l'allemand à l'école primaire a été supprimé à titre provisoire en vue de permettre au français de regagner le terrain perdu pendant l'occupation. Si cette décision n'a pas provoqué de réaction immédiate en raison des événements récents, le Conseil général du Bas-Rhin a néanmoins demandé dès 1946, la réintroduction de l'allemand dans l'enseignement primaire. En 1950, le Conseil général du Haut-Rhin a également sollicité la reprise de l'enseignement de l'allemand dans les écoles alsaciennes, à l'instar d'autres collectivités et instances représentatives.

10 Mais malgré ces prises de position favorables à la réintroduction de l'enseignement de l'allemand à l'école primaire, aucune décision dans ce sens ne fut prise pour éviter de réduire l'enseignement du français.

11 Le milieu syndical enseignant s'est également prononcé contre cet enseignement à l'école, mais les parlementaires ne sont pas restés inactifs et ont présenté plusieurs propositions à l'Assemblée nationale.

12 En 1951, la loi Deixonne relative à l'enseignement des langues et dialectes locaux a prévu une application au breton, au basque, au catalan et à la langue occitane, mais non au corse, au flamand ou à l'allemand en Alsace et en Moselle.

13 En 1952, le décret ministériel du 18 décembre a institué un enseignement facultatif de la langue allemande dans les écoles élémentaires des communes dont la langue usuelle était le dialecte alsacien. L'arrêté du 19 décembre 1952 précisant les modalités d'application du décret prévoyait les dispositions suivantes :

- deux heures d'allemand par semaine durant les deux dernières années de la scolarité obligatoire qui était fixée à 14 ans à l'époque ;

- les deux heures devaient être comprises dans l'horaire obligatoire, dans les limites suivantes : une heure prise sur le temps consacré aux activités diverses, une heure prise sur le temps consacré à l'éducation physique ;

- le volontariat des familles;

- le volontariat des maitres qui devaient se déclarer aptes et disposés à assumer cet enseignement. 

familles en janvier 1953. Cette consultation a donné les résultats suivants :

- 45,1\% des instituteurs ont déclaré accepter d'enseigner l'allemand et 51,7\% ont déclaré refuser cet enseignement ;

- $84,4 \%$ des parents ont souhaité l'enseignement de l'allemand pour leurs enfants et 15,6\% l'ont refusé.

Le refus d'un grand nombre d'instituteurs d'enseigner l'allemand a rendu difficile sa mise en œuvre pour tous les élèves dont les parents se sont déclarés favorables. Cette situation a provoqué des réactions critiques au sein des milieux politiques alsaciens.

Devant la pression des élus, le gouvernement s'est vu dans l'obligation d'agir. Lors d'une audience accordée par le ministre de l'Éducation nationale à une délégation de parlementaires alsaciens, il fut décidé de créer un corps d'instituteurs itinérants pour assurer l'enseignement de l'allemand dans les communes qui n'en bénéficiaient pas.

En 1953, cette décision a été rendue effective par une circulaire du 20 octobre qui a permis la nomination d'instituteurs remplaçants mis à la disposition des inspecteurs primaires qui ont établi des itinéraires de tournées en vue d'assurer le service d'enseignement de l'allemand. Mais, sur le plan pédagogique, ce type de fonctionnement ne semble pas avoir été tout à fait satisfaisant.

De leur côté les élus départementaux ont continué à demander un renforcement de cet enseignement; parallèlement, une commission d'enquête chargée d'étudier les conditions d'application du décret du 18 décembre 1952 et l'opportunité d'apporter des aménagements aux dispositions de ce texte a été instituée. Les propositions de cette commission aboutirent, en janvier 1961, à l'extension de l'enseignement de l'allemand aux trois années terminales des écoles. Mais, ces classes terminales de l'école primaire concernaient exclusivement les élèves âgés de 11 à 14 ans qui n'étaient pas scolarisés dans le cycle secondaire.

19 En 1972, l'inspecteur général d'allemand Georges Holderith a obtenu la réintroduction de l'enseignement de l'allemand dans trente-trois cours moyen première année de l'académie de Strasbourg, à titre expérimental. Cet enseignement a été étendu par la suite à l'ensemble des deux dernières années d'école élémentaire et a été mis en place dans $80 \%$ des classes alsaciennes. Tout enfant dont les parents le souhaitaient devait pouvoir bénéficier de cet enseignement structuro-global audiovisuel qui était dispensé à raison de trente minutes par jour, cinq fois par semaine.

En 1982, une circulaire rectorale sur la langue et la culture régionales en Alsace a précisé pour la première fois que les dialectes alsaciens avaient pour expression écrite une langue de culture et de diffusion internationale : l'allemand. Les textes académiques qui ont été appliqués jusqu'en 1990 ont confirmé cet enseignement précoce de l'allemand en le rendant possible dès le cours élémentaire première année.

21 En 1991, une circulaire rectorale du 20 septembre a défini un programme à moyen terme de développement, de l'allemand à l'école autour de trois objectifs principaux :

- la généralisation effective de l'enseignement précoce de l'allemand dans le dernier cycle de l'école primaire (CE2, CM1, CM2) à raison de trois heures par semaine ;

- l'extension progressive de cette initiation précoce à l'ensemble du cycle précédent en tenant compte des conditions locales, notamment de l'engagement des maîtres ; 
- l'accroissement de l'immersion linguistique et culturelle des élèves par la mise en œuvre de modules d'enseignement en allemand d'un volume horaire hebdomadaire de trois heures, de telle sorte que les élèves de ces classes soient mis en présence active de la langue allemande durant six heures par semaine. grand d'éléments français dans les parlers dialectaux est également un fait linguistique 
qui mérite d'être cité et qui constitue, en fait, une étape du recul du dialecte alsacien qui se fragmente par manque de vocabulaire et qui perd ainsi de sa créativité linguistique.

En Alsace, la situation de la dialectophonie est complexe et variable en fonction des individus, de l'âge, de la catégorie socioprofessionnelle, du milieu urbain ou rural, ou encore du secteur géographique: les statistiques les plus récentes montrent que le dialecte est devenu extrêmement minoritaire dans les grandes villes alsaciennes, mais elles montrent aussi que le dialecte est encore conservé dans certaines zones rurales.

Ce recul dialectal semble d'ailleurs s'accompagner, dans la conscience populaire, d'une perception de plus en plus floue de l'unité qui existe entre l'alsacien et l'allemand. Néanmoins, il faut rappeler que la grande tradition littéraire de l'Alsace est de langue allemande et qu'il serait restrictif de ne parler que de la langue du voisin lorsqu'il existe toujours une littérature germanophone en Alsace. Le bilinguisme scolaire, tel qu'il est développé en Alsace, devrait être susceptible d'atténuer cette courbe descendante; en effet, si l'on considère que la relation de l'allemand dialectal et de l'allemand standard s'inscrit dans un courant circulaire où la connaissance de l'alsacien facilite celle de l'allemand et inversement, l'enseignement bilingue français/allemand devrait avoir des répercussions positives sur la survie de l'alsacien.

En Alsace, l'allemand est langue de proximité et langue régionale : la double dimension de la langue régionale peut donc être considérée comme le ferment d'une identité alsacienne aux ressources multiples qui vont de la culture à l'économie. Dans sa démarche, l'élève d'une classe bilingue traduit rarement, chaque langue étant utilisée de façon directe. Pour le passage d'une langue à l'autre, les facteurs de déclenchement les plus courants semblent être liés à la personne partenaire, à la langue du partenaire et à l'environnement.

Dès l'âge de trois ans, à l'école maternelle, il apparaît que les enfants commencent à prendre conscience du bilinguisme qui se pratique à l'école. D'une manière globale, la compréhension s'installe progressivement et s'acquiert par l'emploi de formes linguistiques voisines et homogènes, notamment dans les rituels et les jeux répétitifs, par la stratégie d'inférence et par l'emploi répété d'autres moyens linguistiques ou non.

Si les champs thématiques sont encore restreints à l'école maternelle, la situation change à partir du cours préparatoire, le développement des contenus d'enseignement des diverses disciplines influant positivement sur la richesse de la thématique. Pour que l'environnement linguistique et culturel des enfants qui fréquentent les sites bilingues ne soit pas artificiel, il est nécessaire de développer le contact des enfants avec la langue allemande authentique autrement que par le seul maitre assurant l'enseignement en allemand.

La prise en compte de la répartition sociolinguistique du français et de l'allemand en Alsace peut se résumer de la manière suivante: le français a le statut de langue officielle ou nationale dans l'espace alsacien et l'allemand joue un rôle important dans le contexte régional. En effet, le contexte régional est le lieu propice à l'application de la politique de développement de l'enseignement bilingue parce qu'il constitue le milieu où les occasions d'interaction sont les plus nombreuses. Dans l'évolution sociolinguistique alsacienne, il apparaît que le caractère urbain s'étend de plus en plus dans la population, alors qu'antérieurement la population rurale et la population urbaine vivaient dans des sphères linguistiques et culturelles distinctes. Ainsi, dans le monde rural, le dialecte et l'allemand standard constituaient les moyens linguistiques privilégiés dans les relations 
courantes professionnelles et à l'église. Le développement de l'urbanisation, la mobilité sociale et géographique ont eu tendance à remplacer progressivement une culture établie et une échelle de valeurs par un modèle national unique ne tenant plus compte de la spécificité alsacienne. Cependant, une prise de conscience linguistique et culturelle tend de nouveau à vouloir développer l'aptitude bilingue et biculturelle de l'Alsace. En fait, le système scolaire alsacien s'adapte progressivement à la demande sociale et le service public de l'Éducation nationale a intégré l'enseignement bilingue français-allemand à parité horaire dans sa mission.

L'attitude des parents, face à cette offre de formation, est généralement très positive parce qu'ils veulent, pour leurs enfants, l'école la mieux adaptée aux besoins de l'environnement social et économique.

Le dispositif d'accompagnement de l'enseignement bilingue prévoit un certain nombre de mesures susceptibles de réaliser un environnement porteur d'authenticité apte à soutenir la motivation des élèves engagés dans la voie de la scolarité bilingue. L'approche double, psychologique et linguistique, conduit les enfants des classes bilingues à reconnaître non plus seulement la langue de l'enseignant de la classe, mais aussi une langue régionale qui est également celle de peuples européens voisins. Sur le plan psychologique, il apparaît que l'image de la langue renvoyée par le milieu environnant est déterminante. De ce point de vue, la parité horaire des deux langues proposée à l'école a un effet positif parce qu'elle permet de déduire qu'il existe une égalité du statut des deux langues.

Le milieu familial joue également un rôle essentiel dans la mesure où il lui incombe d'aider l'enfant à appréhender la situation bilingue scolaire propre à l'Alsace, situation dans laquelle, en majorité, les enfants ne sont exposés à la langue allemande qu'à l'école, les familles doivent relayer l'institution scolaire pour valoriser cette langue. La représentation que l'enfant peut avoir de l'allemand est primordiale pour l'apprentissage de cette langue.

Le modèle alsacien de bilinguisme scolaire est en fait un vecteur d'inter culturalité franco-allemande, dans la mesure où le substrat linguistique et culturel alsacien constitue un trait d'union entre les deux cultures; il s'agit, en l'occurrence, de prendre en compte l'environnement linguistique et culturel concret des apprenants. Ainsi, le sujet bilingue a la possibilité de faire appel à deux codes sémantiques et à deux codes culturels, ce qui lui confère une souplesse d'adaptation et une ouverture à ce qui est autre ou étranger. Le bilingue est en quelque sorte un individu nouveau animé d'une psychologie interculturelle et capable de devenir un médiateur inter linguistique et interculturel.

Par son histoire, par sa situation géographique et linguistique, l'espace alsacien est le lieu où la langue française et la langue allemande sont en contact. Cette spécificité a été prise en compte par les autorités scolaires ainsi que par les collectivités territoriales en Alsace et elle se traduit par la mise en œuvre d'un programme ambitieux de développement de l'enseignement de l'allemand. La question du bilinguisme revêt d'ailleurs une dimension transfrontalière dans la région du Rhin supérieur où la démarche alsacienne tient lieu d'exemple. Si l'on peut parler d'un modèle de bilinguisme alsacien, un modèle bilingue qui porte en lui le ferment d'une meilleure connaissance et d'une meilleure compréhension réciproques, on peut également penser que cette dynamique pourrait être la propédeutique d'un mouvement européen, en vue de trouver un terrain propice à la construction plus solide d'un devenir commun. 


\section{RÉSUMÉS}

La mise en œuvre d'un programme ambitieux de développement de l'enseignement de l'allemand, dès la maternelle, se justifie par le contexte linguistique et historique de la région alsacienne, cette politique pouvant constituer le ferment d'une meilleure connaissance et d'une meilleure compréhension réciproques.

INDEX

Index géographique : France

Mots-clés : enseignement des langues, enseignement bilingue, langue allemande, apprentissage des langues, classe bilingue

\section{AUTEUR}

\section{ROGER SCHUMACHER}

Chargé de mission aux enseignements régionaux et internationaux, Inspection académique du Haut-Rhin, Colmar, France 